\title{
Ab Initio Studies on Hematite Surface and the Adsorption of Phosphate
}

\author{
Swati Chaudhury, ${ }^{1}$ Chandrika Varadachari, ${ }^{1}$ and Kunal Ghosh ${ }^{2}$ \\ ${ }^{1}$ Raman Centre for Applied and Interdisciplinary Sciences, 16A Jheel Road, Kolkata, West Bengal 700075, India \\ ${ }^{2}$ Department of Agricultural Chemistry and Soil Science, University of Calcutta, 35 BC Road, Kolkata, West Bengal 700019, India \\ Correspondence should be addressed to Swati Chaudhury; schaudhury9@gmail.com
}

Received 4 March 2014; Accepted 7 September 2014; Published 28 September 2014

Academic Editor: Jorge M. Seminario

Copyright (C) 2014 Swati Chaudhury et al. This is an open access article distributed under the Creative Commons Attribution License, which permits unrestricted use, distribution, and reproduction in any medium, provided the original work is properly cited.

This investigation explores the ab initio DFT method for understanding surface structure of hematite and the nature and energetics of phosphate adsorption. Using the full potential linearized plane wave method (FP-LAPW), we derived the structure and energies of various magnetic forms of hematite. The antiferromagnetic (AFM) form was observed to be the most stable. Hematite surfaces with Fe-termination, O-termination, or $\mathrm{OH}$-termination were studied. The $\mathrm{OH}$-terminated surface was the most stable. Stability of hematite surfaces follows the order $\mathrm{OH}$-termination $>$ Fe-termination $>\mathrm{O}$-termination. Thus, surface reaction with hematite would occur with the $\mathrm{OH}$ at the surface and not with $\mathrm{Fe}$ atoms. The structure of phosphate adsorbed on hematite was derived. Bonding is through the $\mathrm{H}$ atom of the $\mathrm{OH}$ at the surface. An alternative mechanism of phosphate adsorption on hematite has been derived. Adsorption energy is high and suggests chemisorption rather than physisorption of phosphate on hematite.

\section{Introduction}

Hematite is ubiquitous in all soils but is predominant in soils of the tropical and subtropical regions. Due to large surface area and high reactivity, hematite influences several physical and chemical properties. Modelling the reactivity of hematite surfaces is, therefore, based on its bulk crystal structure and particularly on the surface arrangement of atoms. Hematite, $\alpha-\mathrm{Fe}_{2} \mathrm{O}_{3}$, belongs to the space group $167 \mathrm{R}-3 \mathrm{c}$ with either two or six formula units in the primitive rhombohedral and in the conventional hexagonal unit cells, respectively. Hexagonal close-packed layers of $\mathrm{O}$ atoms are present, with Fe atoms filling 2/3rd of the octahedral holes, which are all in a high-spin $\mathrm{d}^{5}$ electronic configuration [1]. The stable phase is antiferromagnetic (AFM) below the Neel temperature, $T_{N}$ at $955 \mathrm{~K}[2]$.

Catti et al. [3] studied the electronic, magnetic, and structural properties of hematite by periodic unrestricted Hartree-Fock method and showed that the band gap is of p-d rather than d-d type, confirming the charge-transferinsulator nature of hematite. Rollmann et al. [2] studied hematite by using density functional theory (DFT) and the generalized gradient approximation (GGA) and observed that the ground state is antiferromagnetic. Analysis of the density of states confirms the strong hybridization between $\mathrm{Fe} 3 \mathrm{~d}$ and $\mathrm{O} 2 \mathrm{p}$ states. Spin-density functional theory was used for the calculation of slab geometry in the surface study of hematite [4]. They observed that, depending on the ambient oxygen partial pressure, either the iron terminated surface or the oxygen terminated surface may be stable under thermal equilibrium. Rohrbach et al. [5] presented a detailed ab initio investigation of the structural, electronic, and magnetic properties of the $\left(\begin{array}{llll}0 & 0 & 0 & 1\end{array}\right)$ surfaces of hematite by GGA and $(\mathrm{GGA}+U)$ approaches. They observed that Oterminated surfaces are energetically unfavourable compared to the Fe-terminated surface. Using DFT methods, Souvi et al. [6] showed that outermost iron atoms of hematite would be almost fully hydroxylated. This would decrease the Lewis acidity of the surface and domination by hydrogen bonds. Experimental studies using crystal truncation rod (CTR) Xray diffraction, however, showed that the unreacted $\alpha-\mathrm{Fe}_{2} \mathrm{O}_{3}$ $\left(\begin{array}{llll}0 & 0 & 0 & 1\end{array}\right)$ surface consists of both an O-layer terminated domain and a hydroxylated Fe-layer terminated domain [7]. Trainor et al. [8] also used CTR XRD to study hematite 
surfaces and observed that surface is dominated by hydroxyls which are either singly or doubly coordinated with Fe.

Studies on adsorption of small molecules by hematite surfaces using DFT methods are now being increasingly used and have been reported to be quite reliable [9]. DFT supercell calculations revealed that, regardless of nature of surface terminations, the adsorption of methyl radical was strong in all cases. Fe vacancy in hematite showed increased reactivity towards $\mathrm{H}_{2} \mathrm{O}$ [10].

Adsorption reactions of phosphate are of particular interest due to its influence on phosphate availability to plants. The mechanisms of adsorption of phosphate on hematite surfaces have been derived mainly from experimental studies. Goldberg and Sposito [11] studied the kinetics of adsorptiondesorption and proposed ligand exchange as the mechanism of phosphate-surface hydroxyl reaction. According to them, hydroxyl ion release and crystallographic calculations provided support for complexation of phosphate ions on the surface. Elzinga and Sparks [12] characterized phosphate adsorption on hematite as a function of $\mathrm{pH}$ and phosphate concentration by ATR-FTIR investigations and suggested that the surface complexes are monoprotonated. Rajan [13] measured phosphate adsorption on oxide surfaces by displacing ligands $\left[\mathrm{M}(\mathrm{OH})_{2}\right.$ or $\left.\mathrm{M}(\mathrm{OH})\right]$ and recording simultaneous reduction in the net surface charges. He inferred that phosphate was adsorbed on the hydrous oxides only and adsorption makes the oxide surface less positive. Barrón and Torrent [14] reported that surface hydroxyl configuration (SHC) of the hematite $\left\{\begin{array}{lll}1 & 0 & 0\end{array}\right\},\left\{\begin{array}{lll}1 & 1 & 0\end{array}\right\},\left\{\begin{array}{lll}0 & 1 & 2\end{array}\right\},\left\{\begin{array}{lll}1 & 0 & 4\end{array}\right\},\left\{\begin{array}{lll}0 & 1 & 8\end{array}\right\},\left\{\begin{array}{lll}1 & 1 & 3\end{array}\right\}$, and $\left\{\begin{array}{lll}0 & 0 & 1\end{array}\right\}$ faces determined the surface properties of oxides. Singly, doubly, and triply coordinated $\mathrm{OH}$ groups are involved in the specific adsorption of phosphate and other ions. Adsorption of phosphate and sulphate adsorption on ferric hydroxide simulated by DFT [15] showed that adsorption was most favored by bidentate, binuclear surface complexes. Phosphate complexes with iron hydroxides were also studied by quantum mechanical calculations which concluded that monoprotonated monodentate complex should be favored [16].

The objective here was to understand the nature of hematite surface that would be involved in surface reactions by theoretical studies of the structure using DFT-LPAW method. The subsequent goal was to study the geometry and energetics of phosphate adsorption on the surface. This involved (a) a derivation of the structure of the most stable magnetic form of hematite, (b) derivation of the most stable surface termination (Fe-termination, O-termination, or $\mathrm{OH}$ termination) followed by derivation of the structure of this surface, and (c) derivation of the structure and orientation of the adsorbed phosphate and the energetics of the adsorption process. All derivations used ab initio methods based on the density functional theory [17] to derive electron densities and subsequent derivation of ground state properties.

\section{Materials and Methods}

2.1. Computational Methodology. The ab initio derivations were done using Wien $2 k$ [18]; for structural visualization,
Xcrysden [19] was used and structure transformation was done by Cryscon [20].

Wien $2 k$ performs electronic structure calculations of solids using density functional theory. Here, Kohn-Sham equations for the ground state density and total energy are solved by the full potential linearized augmented plane wave (FP-LAPW) method [18] by introducing a basis set which is especially adapted to the problem [21]. Kohn-Sham equations are solved self-consistently in an iterative process under the generalized gradient approximation with the Perdew-BurkeErnzerhof functional for the exchange-correlation energy, subject to periodic lattice boundary conditions [18].

2.2. Geometry and Magnetic Nature of Unit Cell. Hematite belongs to the trigonal space group and can exist in three different magnetic forms, namely, nonmagnetic (NM), spinpolarized (SP), and antiferromagnetic (AFM). The rhombohedral structure was used for ground state calculations. Initially optimization of input parameters, $k$-points, and $R k_{\max }$ was done. $k$-points were studied over 200 to 1400 and observed to be optimum at $1400 . R k_{\max }$ value was optimum at 8.00. Energy cut-off between core and semicore was at -9.0 Ry. All three forms of hematite were optimized for volumes, $c / a$ ratios, and atom positions. Total energies were derived for each of the optimized structures.

2.3. Supercell Calculations and Slab Geometry. Supercell calculations were done with the AFM form of hematite. The rhombohedral cell was transformed into a hexagonal cell using Cryscon. In the hexagonal cell, the $\mathrm{O}$ atoms approximately form a hexagonal close packing (hcp) lattice with $\mathrm{Fe}$ atoms occupying two-thirds of the octahedrally coordinated interstitial positions. The structure has a $-\mathrm{O}-\mathrm{Fe}-\mathrm{Fe}-\mathrm{O}-\mathrm{Fe}-$ Fe-stacking sequence in the $\left[\begin{array}{llll}0 & 0 & 0 & 1\end{array}\right]$ direction, with $\sim 2.20 \AA$ between the $O$ planes in the bulk configuration [5]. Here, $\left(\begin{array}{llll}0 & 0 & 0 & 1\end{array}\right)$ plane was studied.

As the first step, an optimum vacuum slab was derived. A $(1 \times 1 \times 1)$ hexagonal supercell was prepared and vacuum was added in the $Z$-direction. Here, the repeated slab model containing on average 12 atomic layers represents the surfaces. The optimum vacuum depth separating the slab in the $\left[\begin{array}{llll}0 & 0 & 0 & 1\end{array}\right]$ direction was derived so that an intersurface interaction was negligible. The model was constructed such that the inversion symmetry was maintained; that is, both surfaces of the slab were identical. We used spin density functional theory calculations for the slab geometry. Optimized vacuum slab distance for AFM-hematite was at 29 Bohr. This agrees well with data obtained by previous workers [22].

Geometry optimization computations were performed in several stages. The surface atoms were relaxed layer by layer while fixing the rest of the system in order to obtain a good convergence. Towards the end of the convergence process, all atoms were relaxed. A modified tetrahedron integration scheme was used to generate the $k$-mesh in the irreducible wedge of the hexagonal Brillouin zone on a special point grid [9]. A $(4 \times 4 \times 1) k$-mesh was required in the final stages of convergence. $K$-mesh optimization followed by $k_{\max }$ optimization was done. The muffin tin radius, $R_{m t}$, used was 
TABLE 1: Derived crystal parameters of various forms of hematite.

\begin{tabular}{|c|c|c|c|}
\hline Parameters & Crystal systems & Derived in this work & Experimental value \\
\hline \multirow{3}{*}{ Cell dimensions $(\AA)$} & (i) $\mathrm{NM}$ & $a=b=4.7405, c=13.6279$ & \multirow{3}{*}{$a=b=5.035^{2}, c=13.72^{2}$} \\
\hline & (ii) $\mathrm{SP}$ & $a=b=4.8058, c=13.2919$ & \\
\hline & (iii) AFM & $a=b=4.9658, c=13.9104$ & \\
\hline \multirow{3}{*}{ Bulk modulus (Gpa) } & (i) $\mathrm{NM}$ & 274.8133 & \multirow{3}{*}{$230^{3}$} \\
\hline & (ii) SP & 255.9203 & \\
\hline & (iii) AFM & 184.5472 & \\
\hline \multirow{3}{*}{ Coefficient of bulk modulus } & (i) $\mathrm{NM}$ & 4.8655 & \multirow{3}{*}{$3.5^{3}$} \\
\hline & (ii) SP & 13.5683 & \\
\hline & (iii) AFM & 5.8852 & \\
\hline \multirow{3}{*}{ Cohesive energy (eV/formula unit) } & (i) $\mathrm{NM}$ & -27.8402 & \multirow{3}{*}{$-25.171^{3}$} \\
\hline & (ii) SP & -28.1210 & \\
\hline & (iii) AFM & -33.4725 & \\
\hline
\end{tabular}

NM: nonmagnetic, SP: spin polarized, and AFM: antiferromagnetic.

$1.70 \AA$ for $\mathrm{Fe}$ and $\mathrm{O}$ atoms and $0.60 \AA$ for $\mathrm{H}$ atoms. The energy cut-off between core and semicore was -9.00 . The hematite surface was treated antiferromagnetically. In AFMhematite surfaces, spin flipping for $\mathrm{Fe}\left(\mathrm{Fe}_{1}\right.$ and $\left.\mathrm{Fe}_{2}\right)$ was done, that is, inverting the spin up and dn (down). Rotationaltranslational matrix was added in AFM-hematite surfaces for the symmetry operation.

After deriving the optimized slab distance, cell optimization was done. For cell optimization, c/a optimization followed by volume optimization was performed [23]. Using the computed lattice parameters in the direction parallel to the $\left(\begin{array}{llll}0 & 0 & 0 & 1\end{array}\right)$ surface, as obtained from bulk calculations, the atomic positions of all atoms in the slab were fully relaxed, resulting in optimized geometries and corresponding total energies.

Three different surface terminated states of hematite were studied. These were Fe-terminated, $\mathrm{O}$-terminated, and $\mathrm{OH}$ terminated. Each of these structures along with the surfaces was optimized for geometry and the most relaxed positions were derived. The total energies of the most relaxed structures were then derived.

2.4. Phosphate Adsorption. To calculate the adsorption energies and other properties of phosphate on the hematite, phosphate molecules were added to the protonated antiferromagnetic surface of the $\alpha-\mathrm{Fe}_{2} \mathrm{O}_{3}\left(\begin{array}{llll}0 & 0 & 0 & 1\end{array}\right)$ on threefold oxygen site with the phosphate molecule located above the $\mathrm{H}$-terminating oxide plane and the $\mathrm{H}-\mathrm{O}$ bond length initially set at $0.130 \mathrm{~nm}$. The phosphate molecules as well as the top $\mathrm{Fe}_{1}-\mathrm{O}_{1}-\mathrm{Fe}_{2}$ layers were then fully relaxed.

Calculations used the $(4 \times 4 \times 1) k$-mesh, $R_{m t}(\mathrm{Fe})=$ $1.60 \AA, R_{m t}(\mathrm{O})=1.10 \AA, R_{m t}(\mathrm{P})=1.50 \AA, R_{m t}(\mathrm{H})=0.60 \AA, 13$ GGA, $k_{\max }=4.00$, and mixing factor 0.01 (BROYD scheme). For symmetry operation, the rotational-translational matrix was

$$
\left(\begin{array}{lll}
1 & 0 & 0 \\
0 & 1 & 0 \\
0 & 0 & 1
\end{array}\right)\left(\begin{array}{l}
1.0 \\
1.0 \\
1.0
\end{array}\right) .
$$

These strained systems contained huge amounts of forces. So the structures were minimized for forces by changing the atomic positions to derive the structure with minimum forces. Densities of states, electron density mappings, and so forth were plotted with both systems after optimizing the structures.

The adsorption energy $\Delta E$ was evaluated as

$$
\left.\Delta E=E_{\text {tot }} \text { (adsorbed) }-E_{\text {tot }} \text { (separated }\right),
$$

where $E_{\text {tot }}$ (adsorbed) is the total calculated energy of the unit cell containing the adsorbed phosphate molecule in its equilibrium position and $E_{\text {tot }}$ (separated) is that of the system at a large separation of the phosphate molecules from the surface. The $E_{\text {tot }}$ (separated) is equal to the sum of the calculated total energies of the partially optimized $\alpha$ - $\mathrm{Fe}_{2} \mathrm{O}_{3}$ slab and a single phosphate molecule alone.

\section{Results and Discussion}

3.1. Cell Structure and Magnetization. Optimized cell dimensions and other cell properties of the three magnetic forms of hematite are shown in Table 1. Data show that the AFM form has the lowest energy and is, therefore, the most stable of all three forms. Stability follows the sequence AFM > $\mathrm{SP}>\mathrm{NM}$. The stability of the AFM form compared to other magnetic forms of hematite has been corroborated by previous workers $[2,3]$. There appears to be an expansion of the cell in the $a / c$-axis from NM to SP to AFM form of hematite. The $c$-axis is largest for AFM and smallest for the SP form. The derived cell parameters of AFM hematite are close to the experimental data (Table 1), namely, $5.035 \AA$ and $13.72 \AA$, respectively [24]. Other workers theoretically derived cell parameters as $5.025 \AA$ and $13.671 \AA$ [4] and $5.007 \AA$ and $13.829 \AA$ [5]. Bulk modulus of NM, SP, and AFM hematite is in good agreement with the theoretical results by Bergermayer et al. [22].

The experimental band gap of hematite, a "Mott-Hubbard insulator", is $2.0 \mathrm{eV}$. The theoretically derived band gap is not more than $0.50 \mathrm{eV}$ due to strong interaction between $3 \mathrm{~d}$ of 


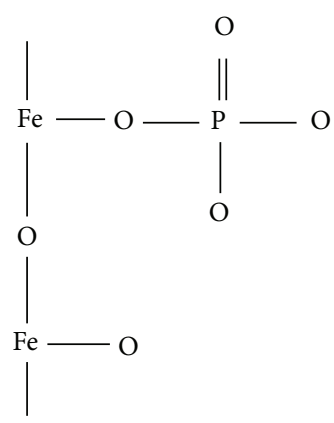

(a)

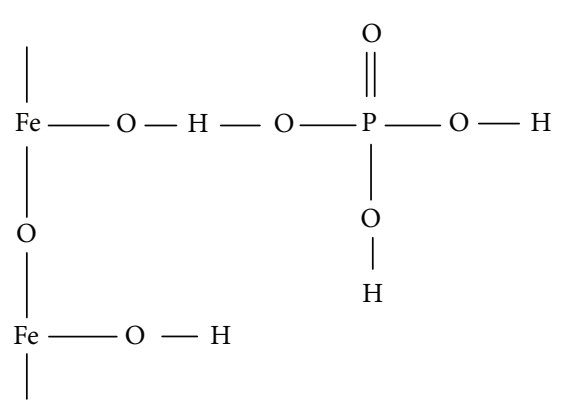

(b)

FIGURE 1: Mechanism of hematite-phosphate interaction. (a) Previous concept [28] (b) proposed concept.

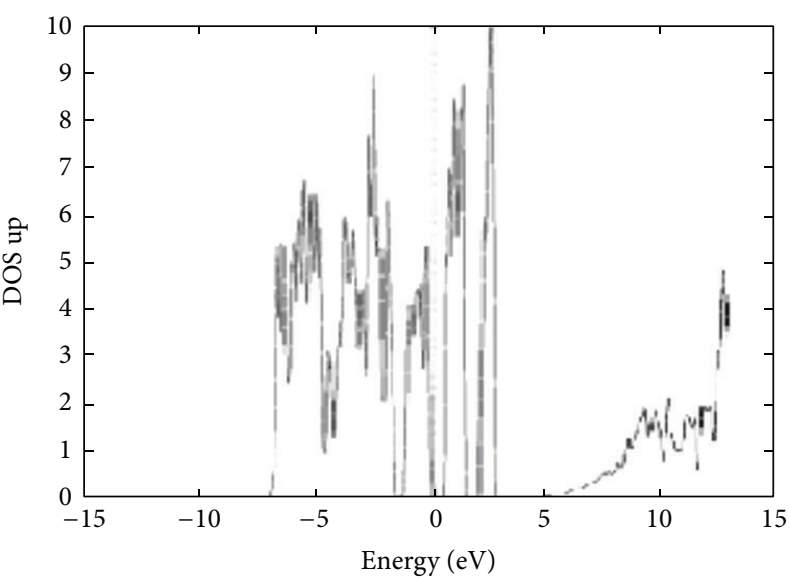

(a)

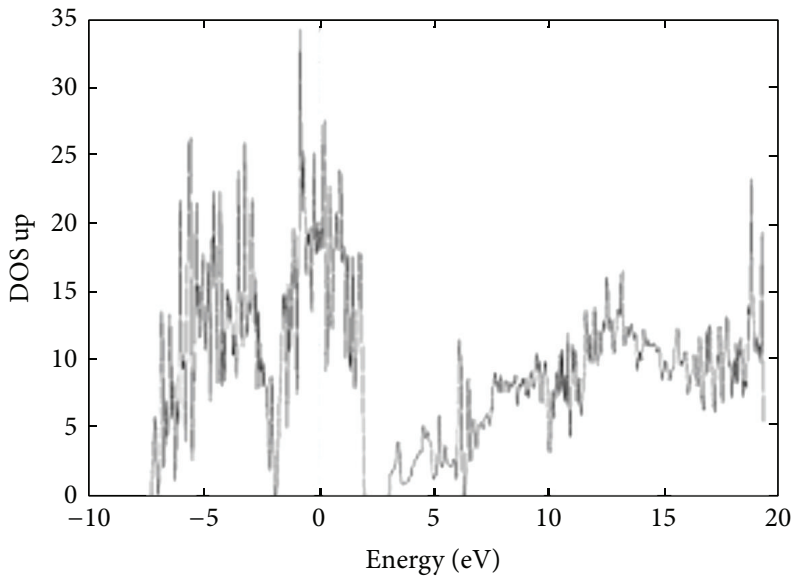

(c)

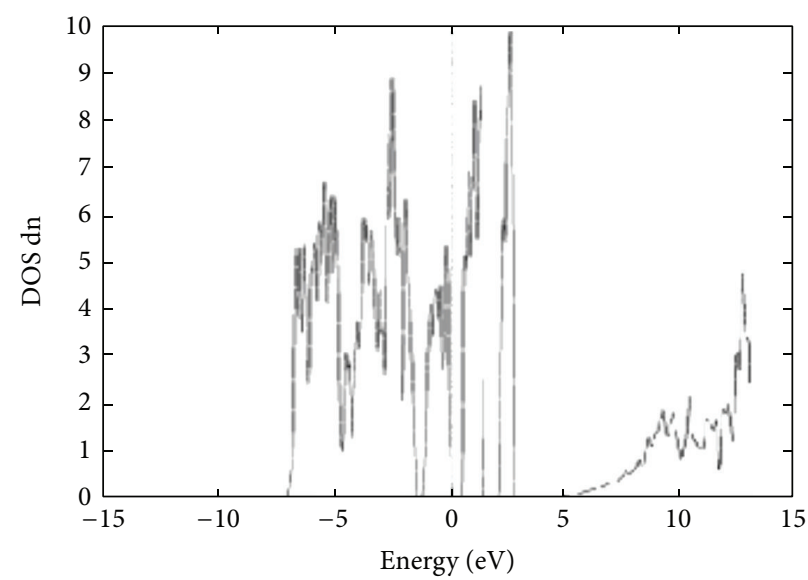

(b)

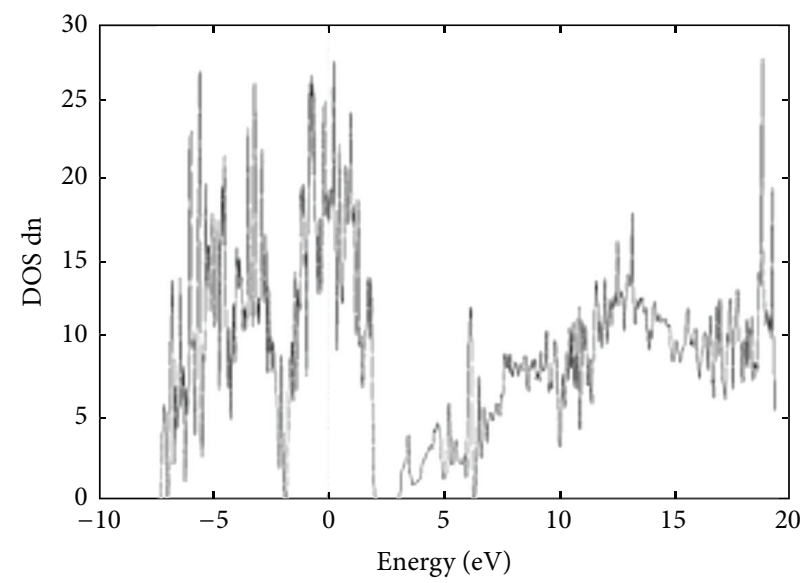

(d)

FIGURE 2: Density of state (DOS) of hematite. (a) up DOS before phosphate adsorption, (b) dn DOS before phosphate adsorption, (c) up DOS after phosphate adsorption, and (d) dn DOS after phosphate adsorption.

Fe and $2 \mathrm{p}$ of oxygen. Since the theory of (DFT + LAPW) cannot deal completely with strongly correlated systems like hematite, $(\mathrm{LDA}+U)$ treatment was required [25]. The value of $U$ used here was $4 \mathrm{eV}$ in ground state calculations of hematite [2]. Applying (LDA + U) on hematite, a band gap of $4.2450 \mathrm{eV}$ and $1.5238 \mathrm{eV}$ was observed for up and dn spins, respectively. The results are in good agreement with those reported earlier [26].

3.2. Surface Stability and Structure. In view of the stability of the AFM form of hematite, surface structural studies were carried out with the AFM hematite. There are basically 


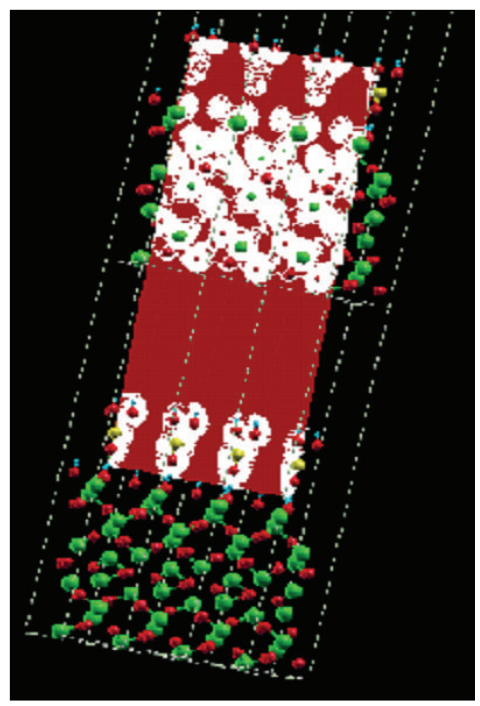

(a)

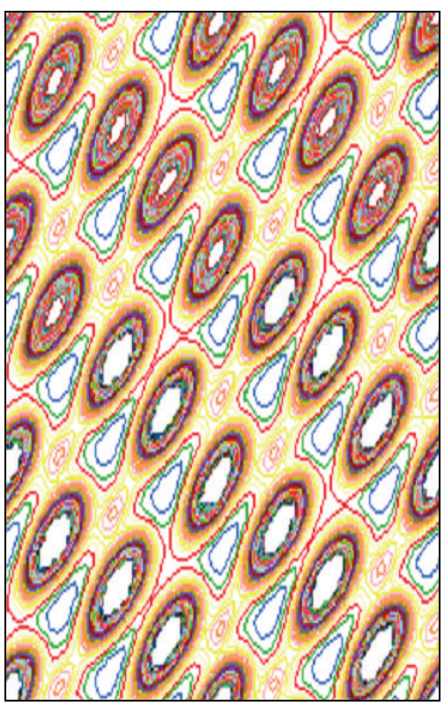

(b)

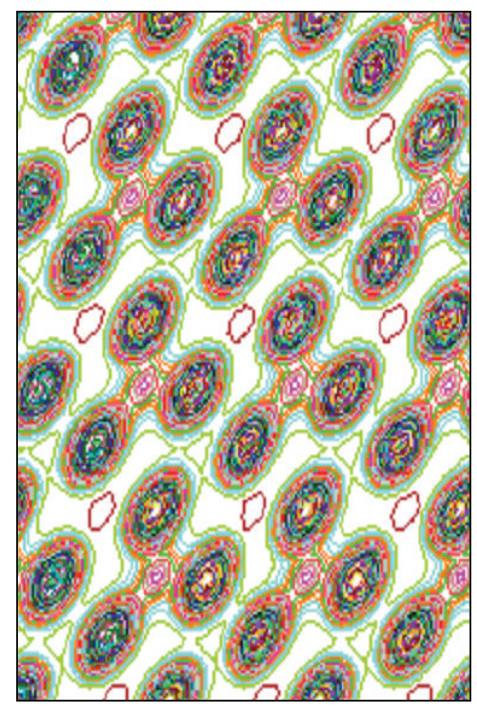

(c)

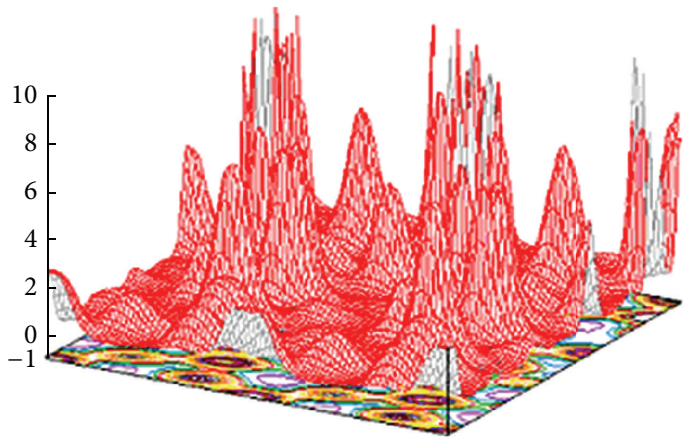

(d)

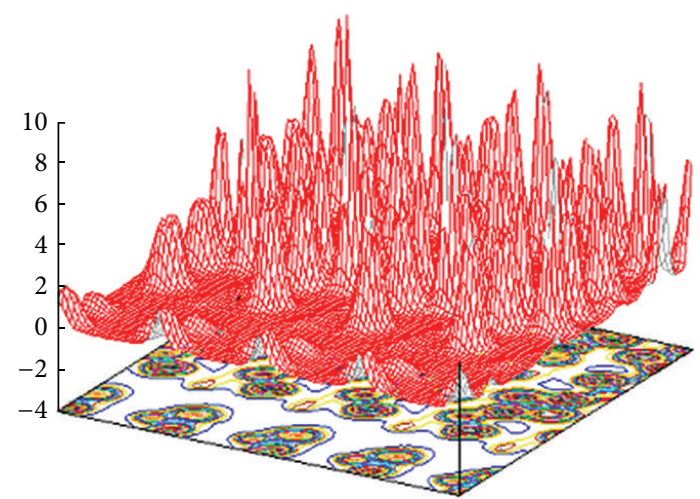

(e)

FIGURE 3: Electron density plots of hematite. (a) Hematite after phosphate adsorption, (b) O-containing plane before phosphate adsorption, (c) O-containing plane after phosphate adsorption, (d) plane containing plane Fe and O before phosphate adsorption, and (e) [3 22 2] plane containing all atoms after phosphate adsorption.

TABLE 2: Stability comparison chart of AFM-hematite.

\begin{tabular}{lc}
\hline Crystal systems & Cohesive energy (Ry/molecule) \\
\hline OH-terminating hematite & -11.7187 \\
Fe-terminating hematite & -11.6393 \\
O-terminating hematite & -9.2695 \\
\hline
\end{tabular}

two ways in which the surface of hematite may terminate, namely, Fe-terminating and $\mathrm{O}$-terminating. However, in the presence of water (moisture from atmosphere), the oxygen terminating surface could get hydroxylated and thus become an $\mathrm{OH}$ surface. Such surfaces would not have dangling bonds. Therefore, for the purpose of these calculations, we also considered the $\mathrm{OH}$-terminating surface.

Cohesive energies of all three terminating forms of hematite are shown in Table 2. The O-terminating surface has highest energy and is, therefore, the least stable; this is followed by the Fe-terminating surface. The $\mathrm{OH}$-terminating surface has the lowest energy of all three forms and hence this is energetically the most stable form of the three. Therefore, the order of stability is $\mathrm{OH}$-terminating hematite $>\mathrm{Fe}$ terminating hematite $>$ O-terminating hematite.

Other workers have also observed that the Fe-terminating surface is more stable than the O-terminating surface. Rohrbach et al. [5] observed that O-terminated surfaces are energetically unfavourable compared to the Fe-terminated surface. Other workers [6] also confirmed the greater stability of Fe-terminated surface over the O-terminating one. Another study has reported that the natural hematite surface consists of singly and doubly protonated layer of oxygen and that the $\mathrm{pK}_{a}$ of the $\mathrm{OH}_{2}$ functional group is approximately 2 [27]. It follows that, in aqueous medium at around neutral $\mathrm{pH}$, the surface $\mathrm{OH}_{2}$ would dissociate to produce $\mathrm{OH}$ groups. This is in conformity with our own derivations. Our finding, on the stability of the $\mathrm{OH}$-terminating surface, is significant because it suggests that surface reactions of hematite occur with the $\mathrm{OH}$ group at the surface and not with surface Fe or O.

3.3. Adsorption of Phosphate. Mechanism of phosphate adsorption is generally viewed as a direct interaction of 
$\mathrm{PO}_{4}{ }^{-}$with the $\mathrm{O}$ at the surface of oxide $[28,29]$, as shown in Figure 1(a). However, our derivations suggest that the stable hematite surface is $\mathrm{OH}$-terminated and not O-terminated. Therefore, the mechanism of adsorption would be by $\mathrm{H}$-bonding through the hydroxylated surface $\mathrm{OH}$ (Figure 1(b)). The adsorption energy for phosphate on hematite is $-9.9716 \times 10^{-18}$ joule/molecule or $-62.2378 \mathrm{eV} /$ molecule. Since the adsorption energy is quite large, the phenomenon is chemisorption and phosphate is not easily available to the plants.

Density of state (DOS) after phosphate adsorption shows remarkable changes for both spin up and dn states. In the total DOS (up), the range for valence band was 2.07 to $-7.23 \mathrm{eV}$ whereas in the DOS (dn) case, the range was 2.07 to $-7.25 \mathrm{eV}$. A small shift in Fermi level is evident. Conduction band range was found to be above $3.05 \mathrm{eV}$ for both total up and dn cases. For phosphate adsorbed hematite (Figures 2(c) and 2(d)), the band gaps were 0.925 and $0.952 \mathrm{eV}$ for up and $\mathrm{dn}$ spins, respectively, whereas the hematite itself showed band gaps of 4.245 and $1.524 \mathrm{eV}$ (Figures 2(a) and 2(b)).

Electron density plots are depicted in Figures 3(a) to 3(e). Electronic distributions around $\mathrm{P}$ and $\mathrm{O}$ of phosphate group are similar due to comparable electronegativity of $\mathrm{P}$ and $\mathrm{O}$. Electronic distribution (Figure 3(e)) in $\mathrm{O}$ atoms shows uneven contribution of electron density from $\mathrm{Fe}$ and $\mathrm{P}$. The extensive charge transfer from the $\mathrm{O}$ to $\mathrm{Fe}$ indicates a predominant ionic bonding resulting in a slightly polarized Fe charge density. This isolated electron distribution of phosphate group from the entire protonated hematite surface proves that the bond formation between phosphate group and hematite surfaces is through hydrogen only.

\section{Conclusion}

The antiferromagnetic (AFM) form has been shown to be the most stable form of hematite. Derivations suggest that the surface of hematite has a layer of $\mathrm{OH}$ and is neither $\mathrm{O}$ terminated nor Fe-terminated. Accordingly, surface reactions occur with the $\mathrm{OH}$ at the surface. Adsorption of phosphate reveals a $\mathrm{Fe}-\mathrm{O}-\mathrm{H}-\mathrm{O}-\mathrm{P}$ mechanism for adsorption rather than a Fe-O-P mechanism. Adsorption energy is high suggesting chemisorption. This can explain the strong retention of phosphate by hematite.

Our study has demonstrated the feasibility of this new approach for reactivity of mineral surfaces. We have shown that it is possible to theoretically derive the surface structure of minerals, which is critical for understanding their reactivity. The geometry of surface adsorbed molecules can be derived and their adsorption energies could be calculated. Thus, reactivity and reaction mechanisms can be theoretically understood. Future work could be undertaken to study other minerals like gibbsite and goethite, using the methodologies that we have initiated here.

\section{Conflict of Interests}

The authors declare that there is no conflict of interests regarding the publication of this paper.

\section{Acknowledgment}

This research was supported by the Raman Centre for Applied and Interdisciplinary Sciences, Kolkata, West Bengal, India.

\section{References}

[1] L. Pauling and S. B. Hendricks, "The crystal structures of hematite and corundum," Journal of the American Chemical Society, vol. 47, no. 3, pp. 781-790, 1925.

[2] G. Rollmann, A. Rohrbach, P. Entel, and J. Hafner, "Firstprinciples calculation of the structure and magnetic phases of hematite," Physical Review B-Condensed Matter and Materials Physics, vol. 69, no. 16, Article ID 165107, 2004.

[3] M. Catti, G. Valerio, and R. Dovesi, "Theoretical study of electronic, magnetic, and structural properties of $\alpha-\mathrm{Fe}_{2} \mathrm{O}_{3}$ (hematite)," Physical Review B, vol. 51, no. 12, pp. 7441-7450, 1995.

[4] X.-G. Wang, W. Weiss, S. K. Shaikhutdinov et al., "The hematite $\left(\alpha-\mathrm{Fe}_{2} \mathrm{O}_{3}\right)$ (0001) surface: evidence for domains of distinct chemistry," Physical Review Letters, vol. 81, no. 5, pp. 1038-1041, 1998.

[5] A. Rohrbach, J. Hafner, and G. Kresse, "Ab initio study of the (0001) surfaces of hematite and chromia: influence of strong electronic correlations," Physical Review B, vol. 70, no. 12, Article ID 125426, 2004.

[6] S. M. O. Souvi, M. Badawi, J.-F. Paul, S. Cristol, and L. Cantrel, "A DFT study of the hematite surface state in the presence of $\mathrm{H}_{2}$, $\mathrm{H}_{2} \mathrm{O}$ and $\mathrm{O}_{2}$," Surface Science, vol. 610, pp. 7-15, 2013.

[7] K. S. Tanwar, S. C. Petitto, S. K. Ghose, P. J. Eng, and T. P. Trainor, "Fe(II) adsorption on hematite ( $\left.\begin{array}{llll}0 & 0 & 0 & 1\end{array}\right), "$ Geochimica et Cosmochimica Acta, vol. 73, no. 15, pp. 4346-4365, 2009.

[8] T. P. Trainor, A. M. Chaka, P. J. Eng et al., "Structure and reactivity of the hydrated hematite (0001) surface," Surface Science, vol. 573, no. 2, pp. 204-224, 2004.

[9] X. Ma, L. Liu, J. Jin, P. C. Stair, and D. E. Ellis, "Experimental and theoretical studies of adsorption of $\mathrm{CH}_{3}$ on $\alpha-\mathrm{Fe}_{2} \mathrm{O}_{3}(00$ 0 1) surfaces," Surface Science, vol. 600, no. 14, pp. 2874-2885, 2006.

[10] S. Yin and D. E. Ellis, " $\mathrm{H}_{2} \mathrm{O}$ adsorption and dissociation on defective hematite (0001) surfaces: a DFT study," Surface Science, vol. 602, no. 12, pp. 2047-2054, 2008.

[11] S. Goldberg and G. Sposito, "On the mechanism of specific phosphate adsorption by hydroxylated mineral surfaces: a review," Communications in Soil Science and Plant Analysis, vol. 16, pp. 801-821, 1985.

[12] E. J. Elzinga and D. L. Sparks, "Phosphate adsorption onto hematite: An in situ ATR-FTIR investigation of the effects of $\mathrm{pH}$ and loading level on the mode of phosphate surface complexation," Journal of Colloid and Interface Science, vol. 308, no. 1, pp. 53-70, 2007.

[13] S. S. S. Rajan, "Changes in net surface charge of hydrous alumina with phosphate adsorption," Nature, vol. 262, no. 5563, pp. 4546, 1976.

[14] V. Barrón and J. Torrent, "Surface hydroxyl configuration of various crystal faces of hematite and goethite," Journal of Colloid and Interface Science, vol. 177, no. 2, pp. 407-410, 1996.

[15] N. Y. Acelas, S. M. Mejia, F. Mondragón, and E. Flórez, "Density functional theory characterization of phosphate and sulfate adsorption on $\mathrm{Fe}$-(hydr)oxide: reactivity, $\mathrm{pH}$ effect, estimation of Gibbs free energies, and topological analysis of hydrogen 
bonds," Computational and Theoretical Chemistry, vol. 1005, pp. 16-24, 2013.

[16] K. D. Kwon and J. D. Kubicki, "Molecular orbital theory study on surface complex structures of phosphates to iron hydroxides: calculation of vibrational frequencies and adsorption energies," Langmuir, vol. 20, no. 21, pp. 9249-9254, 2004.

[17] W. Kohn and L. J. Sham, "Self-consistent equations including exchange and correlation effects," Physical Review, vol. 140, pp. A1133-A1138, 1965.

[18] P. Blaha, K. Schwarz, and J. Luitz, WIEN2k, A Full Potential Linearized Augmented Plane Wave Package for Calculating Crystal Properties, Karlheinz Schwarz, Technische Universitat Wien, Vienna, Austria, 1999.

[19] A. Kokalj, "Computer graphics and graphical user interfaces as tools in simulations of matter at the atomic scale," Computational Materials Science, vol. 28, no. 2, pp. 155-168, 2003.

[20] E. Dowty, Cryscon Version 1.2.1, Shape Software, Kingsport, Tenn, USA, 2006.

[21] R. M. Martin, Electronic Structure: Basic Theory and Practical Methods, Cambridge University Press, Cambridge, UK, 2004.

[22] W. Bergermayer, H. Schweiger, and E. Wimmer, "Ab initio thermodynamics of oxide surfaces: $\mathrm{O}_{2}$ on $\mathrm{Fe}_{2} \mathrm{O}_{3}(0001)$," Physical Review B-Condensed Matter and Materials Physics, vol. 69, no. 19, Article ID 195409, 2004.

[23] S. Cottenier, Density Functional Theory and Family of (L)APWMethods: A Step-by-Step Introduction, 2004.

[24] G. Rohrer, Structure and Bonding in Crystalline Materials, Cambridge University Press, New York, NY, USA, 2001.

[25] A. I. Liechtenstein, V. I. Anisimov, and J. Zaanen, "Densityfunctional theory and strong interactions: orbital ordering in Mott-Hubbard insulators," Physical Review B, vol. 52, no. 8, pp. R5467-R5470, 1995.

[26] J. Velev, A. Bandyopadhyay, W. H. Butler, and S. Sarker, "Electronic and magnetic structure of transition-metal-doped $\alpha$-hematite," Physical Review B: Condensed Matter and Materials Physics, vol. 71, no. 20, Article ID 205208, 2005.

[27] C. S. Lo, K. S. Tanwar, A. M. Chaka, and T. P. Trainor, "Density functional theory study of the clean and hydrated hematite (11̄02) surfaces," Physical Review B, vol. 75, Article ID 075425, 2007.

[28] D. J. Greenland and M. H. B. Hayes, Eds., The Chemistry of Soil Constituents, John Wiley \& Sons, New York, NY, USA, 1978.

[29] S. Goldberg and G. Sposito, "On the mechanism of specific phosphate adsorption by hydroxylated mineral surfaces: a review," Communications in Soil Science and Plant Analysis, vol. 16, no. 8, pp. 801-821, 1985. 

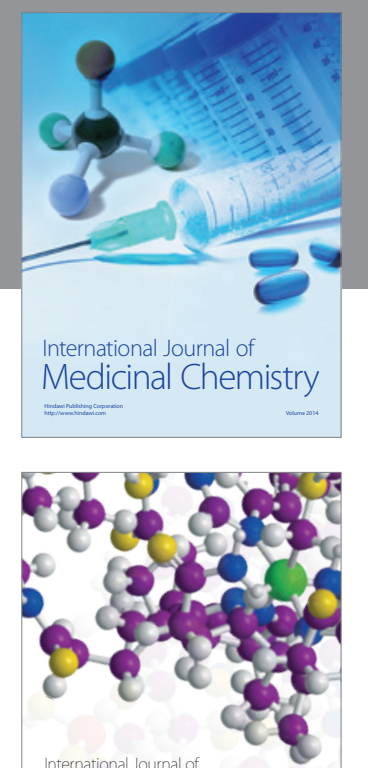

\section{Carbohydrate} Chemistry

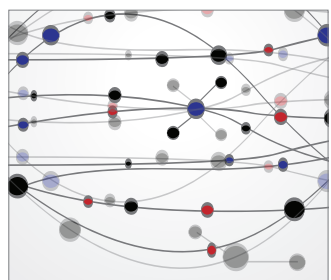

The Scientific World Journal
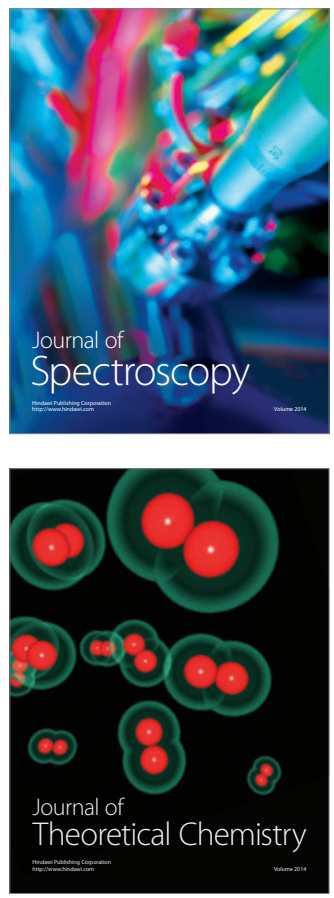
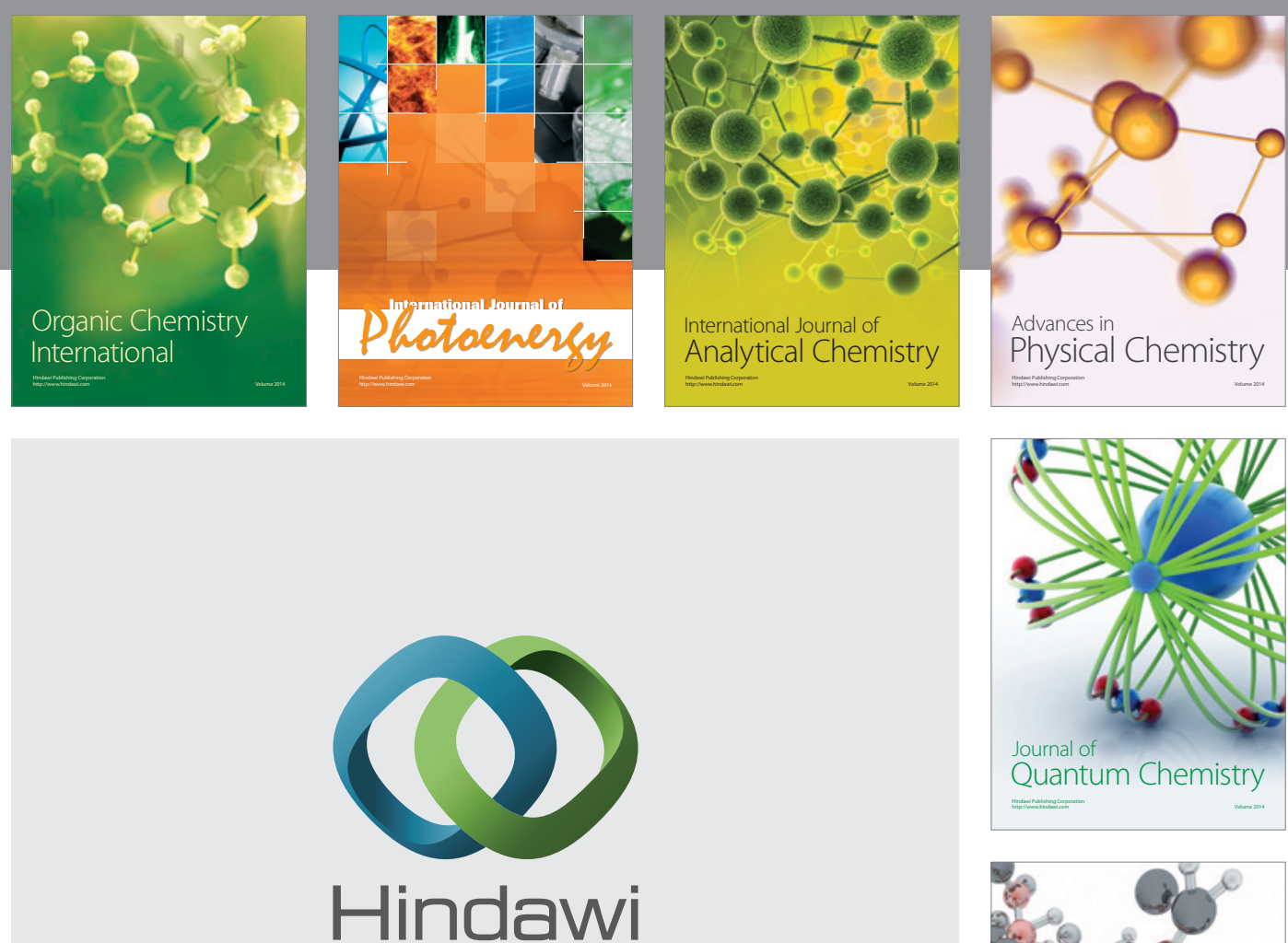

Submit your manuscripts at

http://www.hindawi.com

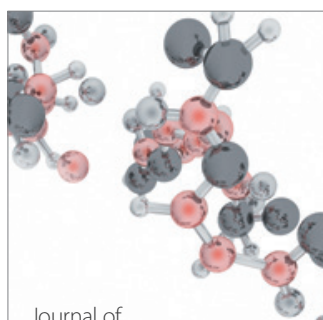

Analytical Methods

in Chemistry

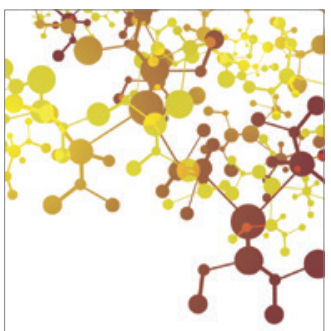

Journal of

Applied Chemistry

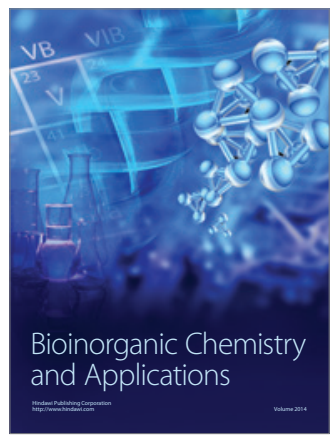

Inorganic Chemistry
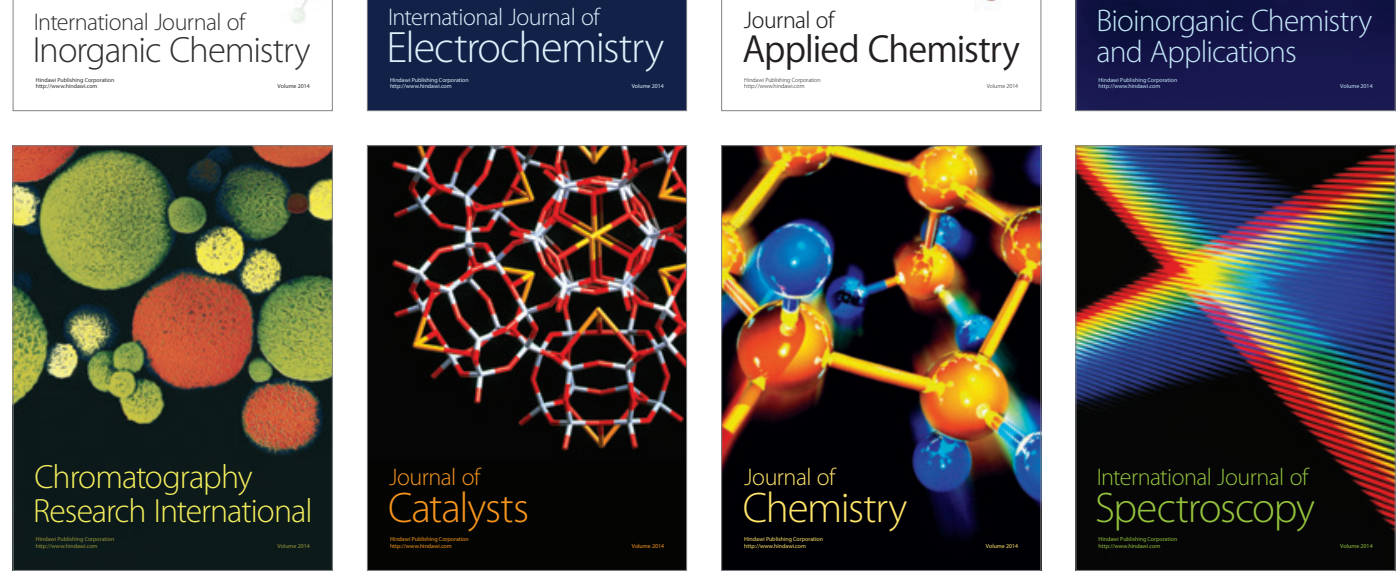